\title{
Diffraction par des réseaux lamellaires multicouches : application à la réalisation d'un polychromateur X-UV
}

K. Krastev*, F. Leguern****, K. Coat*, R. Barchewitz*, J.-M. André*, M.-F. Ravet***, E. Cambril***, F. Rousseaux*** et P. Davi****

* Laboratoire de Chimie Physique, Groupe Optique X, Université Pierre et Marie Curie, CNRS UA 176, 11 rue Pierre et Marie Curie, 75231 Paris cedex 05, France ** CEA-DAM, Service CEM, 91680 Bruyères-le-Châtel, France *** CNRS-L2M, 196 avenue H. Ravera, 92220 Bagneux, France **** SFIM, 14 rue P. Dautier, 78140 Vélizy-Villacoublay, France

Résumé: Le principe de la diffraction par un réseau lamellaire gravé dans miroir multicouche est exposé. Un tel réseau a été réalisé en combinant les techniques de dépôt de couches minces, de lithographie électronique et de "gravure plasma". Une application originale de ce dispositif, appelée "polychromateur", est présentée.

\section{RESEAUX LAMELLAIRES GRAVES DANS UNE STRUCTURE MULTICOUCHE}

Les réseaux lamellaires gravés dans des structures multicouches constituent un système doublement périodique présentant une périodicité en profondeur correspondant à l'empilement multicouche et une périodicité latérale correspondant à la gravure. Un réseau lamellaire est susceptible de diffracter un rayonnement de longueur d'onde $\lambda$ incident perpendiculairement aux traits lorsque les conditions scalaires de Laue suivantes sont satisfaites simultanément (voir figure 1):

$$
\begin{aligned}
& \sin \theta+\sin \theta_{0}=m \frac{\lambda}{d} \\
& \cos \theta-\cos \theta_{0}=p \frac{\lambda}{D}
\end{aligned}
$$

Les angles $\theta_{0}$ et $\theta$ sont respectivement les angles d'attaque et de détection. $d$ et $D$ les périodes de la multicouche et du réseau; $m$ est l'ordre de diffraction de la multicouche et $p$ celui du réseau. En combinant (1) et (2), on en déduit la loi de Bragg généralisée:

$$
\sin \theta_{0}\left[1+\sqrt{1-2 \frac{\cos \theta_{0}}{\sin ^{2} \theta_{0}} \mathrm{p} \frac{\lambda}{\mathrm{D}}-\frac{1}{\sin ^{2} \theta_{0}}\left(\mathrm{p} \frac{\lambda}{\mathrm{D}}\right)^{2}}\right]=\mathrm{m} \frac{\lambda}{\mathrm{d}}
$$

Figure 1: Principe de la diffrnction par un réseau lamellaire d’amplitude gravé dans une suructure multicouche. 


\section{POLYCHROMATEUR X-UV}

Cette dépendance de l'efficacité de diffraction vis à vis de $\lambda, \theta_{0}$ et p peut être exploitée pour concevoir un "polychromateur": pour un rayonnement incident polychromatique, on conçoit que pour $\theta_{0}$ fixé. la relation (3) peut être satisfaite pour plusieurs couples $(\lambda, p)$. Chaque faisceau émergeant à un angle $\theta p$ correspond a l'ordre $p$ et à longueur d'onde $\lambda p$ donnée par:

$$
\lambda_{\mathrm{p}}=2\left[\frac{\mathrm{m}}{\mathrm{d}} \sin \theta_{0} \cdot \frac{\mathrm{p}}{\mathrm{D}} \cos \theta_{0}\right] /\left(\left(\frac{\mathrm{m}}{\mathrm{d}}\right)^{2}+\left(\frac{\mathrm{p}}{\mathrm{D}}\right)^{2}\right]
$$

Ce polychromateur fonctionnant à incidence fixe peut être considéré comme un système qui échantillonne la distribution spectrale du rayonnement incident à un intervalle de longueur d'onde

$$
\delta \lambda \text { donné par: } \quad \delta \lambda=\frac{2 \mathrm{~d}^{2} \cos \theta_{0}}{\mathrm{~m}^{2} \mathrm{D}}
$$

Nous avons utilisé ce type d'échantillonneur pour analyser le spectre d'un tube à rayons $X$ équipé d'une cible de $\mathrm{Cu}$, au voisinage des émissions $\mathrm{Cu}-\mathrm{L}_{\alpha_{\beta} \beta}$. La structure multicouche est constituée d'un empilement de 115 bicouches $W / B_{4} C$ de période $d=3,1 \mathrm{~nm}$ gravé avec une période $\mathrm{D}$ de $1,33 \mu \mathrm{m}$. et un rapport cyclique $\Gamma$ de 0,1 . La figure 2 donne un exemple de la réponse de ce polychromateur pour la source décrite ci-dessus, avec un angle d'attaque fixé à $11,5^{\circ}$

Un tel système peut se révéler intéressant comme séparateur de faisceau ou comme échantillonneur spectral pour source impulsionneile car il ne nécéssite pas de déplacement mécanique au cours de l'acquisition.

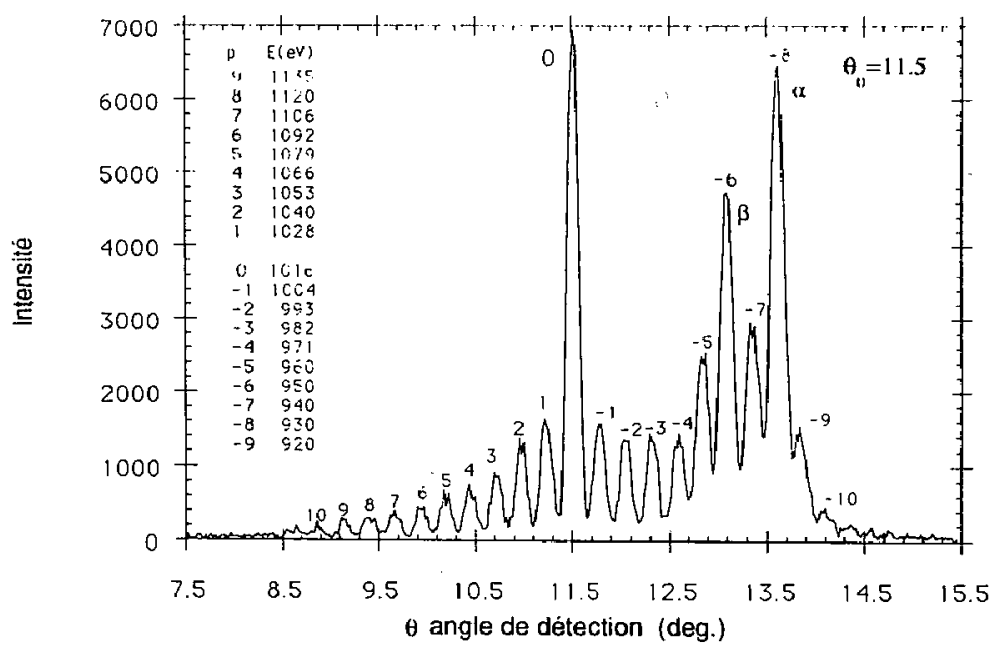

Figure 2: Enregistrement de la réponse du "polychromareur" dont les caractéristiques sont données dans le texte. La source de rayonnement analysée est celle d'un tube à rayons $X$ équipé d'une anode de cuivre. L'analyse est réalisée dans la région des émissions $C u-L \alpha, \beta$. L'angle d'attaque est fixé à $11,5^{\circ}$. On a indiqué en encart les ordres $p$ et l'énergie correspondante: $\alpha$ et $\beta$ correspondent respectivement aux émissions $\mathrm{Cu}-\mathrm{L} \alpha$ et $\mathrm{Cu}-\mathrm{L} \beta$ 